\title{
Changes in Skin Allergy Testing Reactivity observed after a Hurricane. Is the Environment Responsible?
}

\author{
Diego Saporta* \\ Associates in ENY \& Allergy PA, 470 North Ave, Elizabeth NJ 07208, USA
}

Received: June 27, 2015; Accepted: August 28, 2015; Published: September 08, 2015

*Corresponding author: Diego Saporta, Associates in ENY \& Allergy PA, 470 North Ave, Elizabeth NJ 07208, USA, Tel: +908-352-6700; E-Mail: saporta.allergy@gmail.com

\begin{abstract}
Absract
Background: Different patterns of skin reactivity were observed while performing intradermal dilutional allergy tests after the year 2011, the year that a severe storm (Hurricane Irene) affected the author's geographical area.

Methods: In order to study these changes, consecutive patient's allergy charts were arranged in two groups: Group A (Pre-hurricane) contained test results obtained from 2005 to 2010. Group B (Posthurricane) contained test results obtained in 2012 and 2013.

Results: Statistical analysis showed that there were clear differences in the test results between pre and post hurricane groups.

More tests were positive and at significantly weaker dilutions in Group B. The wheal diameters (and spread) were also larger. Group B had an increased number of patients with lower respiratory symptoms and a significant increase in the number of children who tested positive for allergy.

Conclusions: Allergic sensitivity, as reflected by increased skin reactivity and increased airway symptoms were significantly increased in Post-hurricane patients. The patient population in Group B appears to represent a more sensitized and potentially sicker population, suggesting that the potential role of severe climatic events should be considered as a contributing factor to allergic sensitization.
\end{abstract}

\section{Introduction}

In order to successfully treat allergy, it is important to properly identify to which allergens the patient is reactive to, by using standard allergy tests. The Intradermal Dilutional Test (IDT) $[1,2]$ is routinely used for the diagnosis and management of allergic conditions.

In August 2011, the Northeast of the US was affected by Hurricane Irene. At that time, vast areas of the outdoors environment and a large part of the infrastructure were affected and/or destroyed by wind and water. While this Hurricane was not an isolated climatic event $[3,4]$ it was the most devastating one to affect our area in more than 20 years.

By late 2011, it became evident that patients were presenting with unusual skin reactions during Intradermal Testing (IDT).
This consisted of more frequent positive reactions, reactions to weaker allergen concentrations and skin wheals with much larger diameters, indicating a degree of increased sensitivity than previously observed. It was also observed that more patients had Lower Respiratory Symptoms (LRS) and younger patients were seeking allergy evaluation and treatment.

It is recognized that environmental pollution [5,6], including climate changes [7-9], facilitates the development of allergic conditions and asthma. This is one of the few papers to document those changes by comparing pre and post hurricane test results. Environmental exposures have been proposed to lead to a malfunction or sensitization of the immunological system favoring the pro-allergic T- helper type 2 (Th2) allergic pathways [10].

\section{Methods}

To evaluate the hypothesis that skin testing reactivity changed after the severe hurricane of August 2011, consecutive allergy charts were gathered and separated, according to testing dates:

a) Tests performed during 2005-2010

b) Tests performed during 2012 and 2013

c) Tests performed during 2011

Since unusual test results began to appear in late 2011 we chose to eliminate results from this year as it could be considered a "transition period" where changes were not yet fully established. Group A served as a control group as all testing in this group was done prior to the hurricane and the reported changes in Group B are compared to the results in Group A.

Data, including date of a test, age, sex, and test results were entered into an Excel spreadsheet. Test results were recorded as diameter in millimeters ( $\mathrm{mm}$ ) of the skin wheals for each dilution. Results were submitted to a statistician for pre and post hurricane test results comparison (See Statistical analysis below). Each chart was assigned a number to assure confidentiality. IRB was obtained from Trinitas Medical Center, 225 Williamson Street, Elizabeth, NJ 07206. 


\section{Intradermal Dilutional Test (IDT)}

A brief discussion on the IDT follows to facilitate understanding for those not familiar with the diagnostic allergy techniques taught by the American Academy of Otolaryngic Allergy (AAOA) [1,2]. Successive serial 5-fold dilutions of each allergenic extract are made and conventionally labeled as Dilution 1 (1:5), Dilution 2 (1:25), Dilution 3 (1:125), Dilution 4 (1:625), Dilution 5 (1:3125) and Dilution 6 (1:15,625). IDT consists of injecting $0.01 \mathrm{~mL}$ of allergen to produce a 4-mm wheal and measuring the diameter of such wheal 10-15 minutes after injection. Allergen tests are compared to a test control consisting of the same volume of normal saline. The test starts by injecting the weakest dilution (\#6) and advancing to more potent dilutions (\#2 or\#1) until finding the first reactive wheal for the tested allergen. The positive test (reactive wheal) is the first wheal that grows a minimum of $2 \mathrm{~mm}$ greater than the control, which is usually $5 \mathrm{~mm}$. Therefore, a test growing to 7 $\mathrm{mm}$ or more is considered positive. Injection of the next stronger dilution elicits a wheal that will be 2 or more mm larger than the previous reactive wheal, (so it will measure $9 \mathrm{~mm}$ or more) therefore showing a continued increased response at the next higher dilution. The first reactive wheal is called the End Point (EP). The wheal resulting from the injection of the next higher dilution is called the Confirmatory Reaction (CR). The EP or first reactive wheal represents the minimal antigen concentration able to elicit a significant skin response. Test injections that yield a response less than $2 \mathrm{~mm}$ larger than the control are considered to be non-reactive allergens. When the tested allergen is not reactive there will be no response to the injection of even a very large concentration (1:25 dilution or 1:5 dilution of the allergenic extract).

The author personally trains and supervises all nursing personnel and is personally involved in the reading of all test results. Strict testing technique parameters are enforced therefore changes in testing technique over time are unlikely.

\section{Management of the patient with lower respiratory symptoms}

It is usually considered safe to begin testing non-asthmatics by injecting the $4^{\text {th }}$ dilution of the allergenic extract. When a patient has symptoms involving the lower airway: cough, dyspnea, chest tightness or wheezing (lower respiratory symptoms or LRS), has used inhalers or nebulizers or has an abnormal spirometry, the test starts at Dilution $6(1: 15,625)$ otherwise the test starts at Dilution 4 (1:625).Therefore, asthmatics are tested beginning at the $6^{\text {th }}$ dilution. As stated, the objective of the IDT is to determine the lowest dose that will elicit a positive skin reaction, usually without triggering systemic symptoms.

\section{Tested allergens}

Allergens were grouped into two panels

Dust and Animal Dander Panel (DD): Dermatophagoides Pteronyssinus (Der p), Dermatophagoides Farinae (Der f), American cockroach, German cockroach, Cat and Dog.
Pollen panel (P): Hickory, Oak, Sycamore, Pigweed, Ragweed, Bahia and Timothy.

Allergens were obtained from the manufacturer as standardized allergens when available (Der p, Der f, Cat, and Timothy), otherwise as weight/volume $[11,12]$.

\section{Patients}

Two hundred charts from patients with or without asthma were included in the study. One hundred consecutive charts with tests obtained from patients tested in 2005 to 2010 were identified as Group A (Pre-Hurricane).One hundred consecutive charts with tests from 2012 and 2013 were identified as Group B (Post-Hurricane).

\section{Analyzed parameters}

Three parameters were used for this analysis:

1) All positive results (End Points or first reactive wheals) (Table 2).

2) Total Average: Average of the diameter of all reactive wheals (RW) in each panel (RW's included all EP's and CR's, so any wheal that grew to a diameter of $7 \mathrm{~mm}$ or larger was included for analysis) (Table 2).

3) Dilution 6: Allergen tests with positive results at Dilution 6 (Table 3)

Analysis consisted of comparing the number of positive reactions, the wheal diameters and diameter variance (See Statistical analysis below).

\section{Statistical analysis}

ANOVA test was used to study the difference in the average of wheal diameters between both the groups. The Welch test was used to evaluate the spread or Variance of the wheal diameters in both the groups (In statistics, Variance is defined as the square of the standard deviation: $\mathrm{V}=\mathrm{STD}^{2}$ ). The Chi-Square test was used to determine if the frequency of the results differed between groups. The evaluation was performed for each of the groups (Group A and B) as well as a comparison of one against the other for the parameters above mentioned.

\section{Test examples}

Below is an example of a test for DD from Group A on the left and from Group B on the right (figure 1). The allergen dilutions are numbered from 6 (weakest) to 1 (strongest). The numbers in the boxes represent the diameter in millimeters $(\mathrm{mm})$ of each wheal: $5 \mathrm{~mm}$ implies a non-reactive wheal, $7 \mathrm{~mm}$ or more represents a reactive wheal. By definition when an allergen is reactive (yields a positive test) the diameter of the wheal is expected to increase 2 or more mm between dilutions. It is extremely unusual to have an increase of more than 3-4 $\mathrm{mm}$ as the concentration increases. As shown in the example, unusual wheal growth or "hyper-reactive responses" were commonly observed in the Post-Hurricane tests.

The EP's are marked with a circle; the hyper-reactive results are marked in bold. In the above example both dust mites and 
Group A (Pre-Hurricane) test example

\begin{tabular}{|l|l|l|l|l|l|l|}
\hline Dust/Dander & $\mathbf{6}$ & $\mathbf{5}$ & $\mathbf{4}$ & $\mathbf{3}$ & $\mathbf{2}$ & $\mathbf{1}$ \\
\hline Der p & 5 & & 5 & 7 & 11 & \\
\hline Der f & 5 & & 5 & 7 & 11 & \\
\hline Cat & 5 & & 5 & & 7 & 9 \\
\hline Dog & 5 & & 5 & & 5 & 7 \\
\hline A Roach & 5 & & 5 & & 5 & 7 \\
\hline G Roach & 5 & & 5 & & 5 & 7 \\
\hline
\end{tabular}

dander panel were reactive and coincidentally the initial level of reactivity (EP) was dilution 3 for both groups. In the Group A example, the wheal growth occurs as expected (5 -7 -11). In the Group B example, the wheal growth is unusual and surpasses the expected pattern of growth: 5 -15-17 and 5-18-25. It was the repeated observation of these hyper reactive results that motivated this analysis.

\section{Results}

Both groups, A \& B, included one hundred cases (Table 1) There is no statistical difference between the groups for age or gender. There is a statistically significant increase in the number of children and number of patients with asthma or LRS in Group B $(P<0.01)$

Although not all the patients were tested for both panels there is no statistical difference in the number of tests run in each group; therefore Groups A \& B are similar in this respect (Table 2). The number of positive EPs was analyzed for each panel in each group. Table 2 shows that the percentage of positive tests in Group B was higher for both panels.

The concept of Total Average (TA) was used to compare the degree of reactivity among all patients by considering all reactive wheals. TA is defined as the average diameter of all the reactive wheals (EP and CR) that grew to $7 \mathrm{~mm}$ or more. TA was used because it was clinically observed that there was a large disparity in the diameters of the wheals obtained during testing in 2012 and 2013 as compared to the hurricane before. Both the average diameter and the diameter variance (square of the standard deviation) were significantly larger for both the panels in Group B $(P<0.001)$ (Table 2).

When considering tests results only at Dilution 6, it was found that in Group B more tests were required to be done at dilution 6 for both DD and P panels combined because more of the posthurricane patients presented with LRS. A higher percentage of those tests were reactive $(P<0.05)$ (Table 3$)$. Both findings indicate increased allergic sensitization of the post-hurricane patient population.

\section{Results Summary}

Group B had a greater preponderance of children $(P<0.01)$ (Table 1), had more patients with LRS $(P<0.01)$ (Table 1), had more positive test results $(P<0.001)$ (Table 2$)$, both the Total Average and Variance of all RW's was larger $(P<0.001)$ (Table
Group B (Post-Hurricane) test example

\begin{tabular}{|l|l|l|l|l|l|l|}
\hline Dust/Dander & $\mathbf{6}$ & $\mathbf{5}$ & $\mathbf{4}$ & $\mathbf{3}$ & $\mathbf{2}$ & $\mathbf{1}$ \\
\hline Der p & & & 5 & $\mathbf{1 5}$ & $\mathbf{1 7}$ & \\
\hline Der f & & & 5 & $\mathbf{1 8}$ & 25 & \\
\hline Cat & & & 7 & 9 & & \\
\hline Dog & & & 5 & & 5 & 7 \\
\hline A Roach & & & 5 & 7 & 9 & \\
\hline G Roach & & & 5 & 7 & 9 & \\
\hline
\end{tabular}

$2)$, the number of patients required to be tested at Dilution 6 $(1: 15,625)$ was larger $(P<0.05)$ (Table 3$)$ and the number of positive reactions at that dilution was significantly larger $(P<$ 0.05) (Table 3).

\section{Conclusion}

Skin reactivity in patients tested in 2012 and 2013 after the Hurricane was significantly increased as compared to patients tested on or before the Hurricane. This is suggested by the presence of larger (hyperreactive), wider and more frequently positive wheals in the skin test.

The patient population presenting with allergy symptoms in Group B appears to represent a more sensitive and potentially sicker population than the Pre-hurricane cohort. This is suggested by the presence of more positive test results in the overall test, more positive results at the weakest dilution (Dilution 6), and the fact that more children and more patients with LRS were seen in the Post-Hurricane group Group B.

The potential role of severe climatic events should be considered as a factor leading to allergic sensitization.

\section{Discussion}

It is not possible to be certain about reasons for the changes here reported, but the potential role of severe climatic events should be considered as a factor leading to allergic sensitization. The increasing prevalence of allergic conditions may be related to the increasing levels of environmental pollution[13]. It is difficult to demonstrate that a specific circumstance in the environment is responsible for a certain change in the level of reactivity when patients are tested. The northeast of the US suffered several storms, two of which are well known for their destructiveness: We first noticed significant changes in our patient population and their hypersensitivity after Hurricane Irene in 2011. Whatever effect this hurricane had, it was amplified by Hurricane Sandy which further brought destruction to our area in 2012.

These storms strongly affected vast areas of the outdoor environment and also affected and/or destroyed a large part of the infrastructure in the eastern part of New Jersey, where the author's office is located. Water damage after hurricanes and floods increases the likelihood of mold contamination in buildings [14] and exposure to fungal contamination can affect the respiratory and other systems [15]. 
Table 1: Demographic information.

\begin{tabular}{|c|c|c|c|c|c|c|}
\hline GROUP & Number of patients & Age \pm STD (range) & Male & Female & $\leq 18$ & LRS \\
\hline A (Pre- Hurricane) & 100 & $38 \pm 21(13-89)$ & 46 & 54 & 6 & 28 \\
\hline B (Post-Hurricane) & 100 & $49 \pm 18(5-80)$ & 36 & 64 & $30 * *$ & $47^{* *}$ \\
\hline
\end{tabular}

A: Tests from 2010 and before

B: Tests from 2012 and 2013

Number of patients: 100 in each Group

Age \pm STD (range): Mean Age with standard deviation and age range

$\leq 18$ : Child 18 years of age or younger

LRS: Lower respiratory symptoms. Includes patients with diagnosis of asthma, symptoms pertaining the lower airway including exercise induced or abnormal spirometry

**: $P<0.01$

All values indicate percentages as the sample has 100 individuals.

Table 2: Number of Tests and Patients in each panel, Positive results and Total average.

\begin{tabular}{|l|l|l|l|l|}
\hline & Group A DD & Group B DD & Group A Pollen & Group B Pollen \\
\hline Number of patients & 99 & 100 & 88 & 100 \\
\hline Number tests & 676 & 699 & 609 & $701^{\#}$ \\
\hline Positive Result (EP) & 448 & $\mathbf{5 4 0}$ & 310 & $\mathbf{4 1 9}$ \\
\hline \% of Total & 66.3 & $\mathbf{7 7 . 3} * *$ & 50.9 & $\mathbf{5 9 . 9} * *$ \\
\hline Total Average & & & & \\
\hline Number of RW's (Total $\mathbf{m m})$ & $800(6714)$ & $\mathbf{7 7 6}(6909)$ & $563(4839)$ & $601(5665)$ \\
\hline Average (mm) & 8.39 & $\mathbf{8 . 9 0 * *}$ & 8.60 & $\mathbf{9 . 4 3} * *$ \\
\hline Variance & 3.57 & $\mathbf{7 . 9 7} * *$ & 5.30 & $\mathbf{1 6 . 2 6} * *$ \\
\hline
\end{tabular}

Number of patients: Number of patients that were tested in each group

Number tests: Total number of skin tests run for each panel in each group

Group A DD: Dust and Dander panel in Group A (tests from 2010 and before or Pre-Hurricane)

Group B DD: Dust and Dander panel in Group B (tests from 2012 and 2013 or Post-Hurricane)

Group A Pollen: Pollen panel in Group A (tests from 2010 and before or Pre-Hurricane)

Group B Pollen: Pollen panel in Group B (tests from 2012 and 2013 or Post-Hurricane)

Positive Results (EP): Number of skin tests that were positive (reactive)

Total average: Average diameter of all reactive wheals

Number of RW's (Total $\mathrm{mm}$ ): Total number of wheals that had $7 \mathrm{~mm}$ or more (Total cumulative diameters)

Variance: Spread of the diameters $\left(\right.$ Variance $=\mathrm{STD}^{2}$ )

$\#: \mathrm{x}^{2}=0.227, \mathrm{p}=\mathrm{N} / \mathrm{S}$ (not significant)

**: $P<0.001$

Table 3: Tests at Dilution 6. Both panels.

\begin{tabular}{|l|c|c|}
\hline DD \& Pollen & Tests Dil 6 & Pos results (\%) \\
\hline Group A & 286 & $21(7.3 \%)$ \\
\hline Group B & $\mathbf{5 9 0}$ & $\mathbf{7 3 ( 1 2 . 4 \% )} * *$ \\
\hline
\end{tabular}

DD \& Pollen: Dust-Dander and Pollen panels

Group A: Pre-Hurricane (2010 and before)

Group B: Post-Hurricane (2012 \& 2013)

Tests Dil 6: All tests performed with the $6^{\text {th }}$ Dilution

Pos results (\%): Number of positive results from the total of individual tests (percentage)

**: $P<0.05$

Environmental pollution [5,6,13], including climate changes [7-9], facilitates the development of allergic conditions and asthma by leading to a malfunction of the immunological system favoring the T- helper type 2 (Th2) allergenic pathway [10]. Hurricane Irene occurred shortly before the time the author started to observe the changes here described. The environmental effects of a hurricane have been well documented. Even though a cause and effect relationship regarding allergy cannot be proven, it is possible that this storm (and Hurricane Sandy which followed 14 months later) have contributed to environmental changes that could explain these findings.

Many other storms have occurred in our area before Irene $[3,4]$. It is possible that these weather-related events are links in 
a long chain of environmental events where patients over time become more reactive to allergens to which they are exposed. If environmental pollution is able to affect the immunological system in such a way that people become more susceptible to develop allergic conditions, it is possible that the population of Eastern New Jersey became a more sensitive and reactive population and a sicker population (with more severe allergic disease like asthma or LRS that may suggest bronchial hyper reactivity).

It is very difficult from this type of study to demonstrate whether present day population is sicker than the population evaluated prior to the hurricane exposure. The following findings in our study suggest this could be the case:

When a patient has asthma or LRS, standard intradermal testing begins at the $6^{\text {th }}$ dilution. Our finding that more Group B patients were tested at Dilution 6 (Table 3) attests to the fact that the post-hurricane patient population was clinically diagnosed to have a higher incidence of upper respiratory symptoms and/or asthma. The fact that a higher percentage of IDT were positive in Group B (Tables 2 \& 3) also supports the hypothesis that a climatic event altered the sensitivity of the population to a variety of allergens. More children tested in Group B and more patients with LRS in this group (Table 1) suggest that we could be witnessing an earlier onset of a more severe disease in posthurricane patients.

Furthermore, finding more positive tests with wheals that had larger diameter and wider spread (Table 2) with higher levels of reactivity (Table 3 ) also suggests that Group B is potentially not only more sensitized but also more reactive than the population from the same patient area as seen before these climatic events.

Published literature supports the possibility that climatic events can affect the environment $[7,8,9]$ and therefore, the health of the population $[10,15]$.

Mold grows in materials that remain wet for more than 48 hours, and flooding, particularly when floodwaters remain for days or weeks, provides an optimal opportunity for mold growth [14]. Increase in asthma incidence has been noted after Hurricane Floyd (that affected North Carolina to New York in 1996) [16]. A strong association between respiratory symptoms and exposure to water-damaged homes has been established after Hurricanes Katrina and Rita (that affected Louisiana in August and September 2005) [17]. Several studies assessed the allergen content of the homes in that area. Some studies were done 1-2 months after the hurricanes [18,19], and some studies were done several months later, while cleanup and remediation efforts had already begun [20-22]. While all these articles agree that wet conditions provided an ideal environment for mold and bacterial growth with subsequent potential health effects, only in the articles that evaluated the affected area 1-2 months after the hurricanes $[18,19]$ was the concentration of indoor mold found to be significantly higher than the mean concentration outdoors.

One study evaluated home allergen concentration as well as skin responses by prick test of the people living in affected homes after Hurricane Katrina [20] but this study had no control data pertaining to the period prior to the hurricane. Those studies, by the nature of their design [18-22 ] did not compare what was found in the aftermath of those natural disasters to that population's health before the hurricanes.

It is known that dampness and the resultant mold growth that ensures have an adverse health effect on the inhabitants of those buildings. It is logical to assume that the flooding and the subsequent mold growth that followed Hurricanes Irene and Sandy could have been a factor in the findings here reported.

Our data offers a unique opportunity to evaluate changes in a population that live in a stable geographical area, that have attended a single allergy clinic and that has been evaluated by the same practitioner using the same testing techniques, thus increasing the chance that these are significant observations.

Through epidemiological studies it has been shown that the prevalence of asthma has increased from $7.3 \%$ in 2001 to $8.4 \%$ in 2010 for adults and from $8.7 \%$ in 2001 to $9.3 \%$ in 2010 for children [23]. While the reasons for this are likely multifactorial, the role of the environment as suggested by this study should be strongly considered. To more definitely answer the question of whether or not a climactic event such as a hurricane can make a population sicker would involve studies involving larger groups of patients belonging to different geographical areas, including not only test results but also symptom-comparison from before and after a major climatic event. Ideally, clinical studies should be conducted in areas that typically are affected by severe storms, not only measuring the amount of allergens in the indoor and outdoor environments but also studying medical records from before and after the storm. While knowing allergen concentration is an important piece of information, not all the individuals will become sensitized in the same way or react in the same way. Studying the symptoms that develop as a consequence of allergic sensitization will concretely give information about this issue.

\section{Other factors that could have contributed to these findings include}

a) Antigen lot-to-lot variation: Theoretically a new batch of allergenic extract could be more (or less) reactive than the previous batch. This could theoretically explain a random variation in test results but not a persistent change in test results in one direction only. Present day, allergenic extract manufacturer companies use sophisticated technology for the preparation of allergenic extracts which minimizes the possibility of large batchto-batch variation.

b) It could be argued that if asthmatic and young children were more reactive, then the presence of this type of patient in Group B would be the reason for the difference in the results between both groups. Given that these patients came from the same geographical patient area, and that the practice has a stable and homogeneous patient base, the sample size is large enough to eliminate the possibility of primarily selecting one type of patient over the other. Rather it is likely that our hypothesis is correct and allergies in the post-hurricane population are developing 
earlier with such severity to warrant parents and patients to seek help sooner.

The data in this report supports the author's impression that something about the hurricane of 2011 caused a significant change in allergen sensitivity in what had been a stable patient population. Years ago it was rare to see so many allergic children or so many asthmatic patients-child or adult. It is now common at the time of the initial consultation to see pediatric patients who have already been diagnosed with asthma, who are or have used nebulizers or have an exercise- induced asthma and/ or the shortness of breath. This is a distinct change in disease presentation when comparing patients from before and after the hurricanes of 2011 and 2012. It has been shown that after an initial negative answer regarding asthma, if the patient is asked more specifically about the presence of symptoms of lower airway involvement, the presence of nighttime symptoms, exercise triggered symptoms or previous use of inhalers, a much higher percentage of patients will admit to having symptoms suggestive of lower airway hyperreactivity [24].

The information presented here should be considered as an observation, but hopefully this information will provide a stimulus for a large population study with the intention of determining if the changes here described are really happening in other populations exposed to hurricanes. Our findings suggest that in addition to the initial destruction of habitat, hurricanes also somehow (perhaps by stimulating increased mold growth and mycotoxin production) seem to sensitize the affected population for increased reactivity to the many allergens in their environment.

\section{Acknowledgments}

Statistical analysis was performed by Yassir M. Samra, Ph.D, Assistant Professor of management, Manhattan College.

\section{References}

1. Mabry RL. Skin End Point Titration, AAOA Monograph Series, New York, USA: Thieme Medical Publishers; 1994.

2. Fornadley JA. Skin testing in the diagnosis of inhalant allergy. In: JH Krouse SJ. Chadwick BR. Gordon and MJ. Derebery, Eds. Allergy and Immunology: An Otolaryngic Approach, Philadelphia, Pa, USA, Lippincott Williams \& Wilkins, 2002. p. 114-123.

3. List of New Jersey hurricanes. From Wikipedia. In: wikipedia.org/ wiki/List_of_New_Jersey_hurricanes. Accessed January 15, 2015.

4. www.weather2000.com/NY_Snowstorms.html. Accessed January 15, 2015.

5. Schildcrout JS, Sheppard L, Lumley T et al. Ambient air pollution and asthma exacerbations in children: an eight-city analysis. Am J Epidemiol. 2006;15;164(6):505-517.

6. Lee SY, Chang YS, Cho SH. Allergic diseases and air pollution. Asia Pacific Allergy 2013;3(3):145-154. doi: 10.5415/apallergy.2013.3.3.145.

7. Pawankar R, Canonica GW, Holgate ST. WAO White Book on Allergy. Milwaukee: World Allergy Organization; 2011. http://www. worldallergy.org/UserFiles/file/WAO-White-Book-on-Allergy_web. pdf.

8. Nasser SM, Pulimood TB. Allergens and thunderstorm asthma. Curr Allergy Asthma Rep. 2009;9(5):384-390.
9. D’Amato G, Baena-Cagnani CE, Cecchi L, Annesi-Maesano I, Nunes C, Ansotegui I, et al. Climate change, air pollution and extreme events leading to increasing prevalence of allergic respiratory diseases. Multidiscip Respir Med. 2013;8(1):12. doi: 10.1186/2049-6958-8-12.

10. Liu J, Ballaney M, Al-alem U, et al. Combined inhaled diesel exhaust particles and allergen exposure alter methylation of $\mathrm{T}$ helper genes and IgE production in vivo. Toxicol Sci. 2008;102(1):76-81.

11. Antigen Laboratories, Inc.30 South Main Street. Liberty MO USA (NOTE: Since this data was collected Antigen Laboratories has been acquired by Greer Laboratories, 639 Nuway Circle NE, Lenoir, NC 28645).

12. Alk-Abello, Inc.1700 Royston Lane, Round Rock, TX, USA.

13. Rea WJ. Chemical Sensitivity. CRC Press 1992.

14. Brandt M, Brown C, Burkhart J, Burton N, Cox-Ganser J, Damon S, et al. Mold prevention strategies and possible health effects in the aftermath of hurricanes and major floods. MMWR Recomm Rep. 2006;55(RR-8):1-27.

15. Metts TA. Addressing environmental health implications of mold exposure after major flooding. AAOHN J.2008;56(3):115-120.

16. Morbidity and mortality associated With Hurricane Floyd--North Carolina, September-October 1999. MMWR Morb Mortal Wkly Rep. 2000;49(17):369-372.

17. Cummings KJ, Cox-Ganser J, Riggs MA, Edwards N, Hobbs GR, Kreiss K. Health Effects of exposure to water-damaged New Orleans Homes six months after Hurricanes Katrina and Rita. Am J Public Health. 2008 May;98(5):869-875. doi: 10.2105/AJPH.2007.118398.

18.Solomon GM, Hjelmroos-Koski M, Rotkin-Ellman M, Hammond SK. Airborne Mold and Endotoxin Concentrations in New Orleans, Louisiana, after Flooding, October through November 2005. Environ Health Perspect. 2006; 114(9):1381-1386.

19. Rao CY, Riggs MA, Chew GL, Muilenberg ML, Thorne PS, Van Sickle D, et al. Characterization of Airborne Molds, Endotoxins, and Glucans in Homes in New Orleans after Hurricanes Katrina and Rita. Appl Environ Microbiol. 2007;73(5):1630-1634. doi: 10.1128/AEM.01973-06.

20. Grimsley LF1, Wildfire J, Lichtveld M, Kennedy S, El-Dahr JM, Chulada PC, et al. Few Associations Found between Mold and Other Allergen Concentrations in the Home versus Skin Sensitivity from Children with Asthma after Hurricane Katrina in the Head-Off Environmental Asthma in Louisiana Study. Int J Pediatr. 2012;2012:427358. doi: 10.1155/2012/427358.

21. Mitchell H1, Cohn RD, Wildfire J, Thornton E, Kennedy S, El-Dahr JM, et al. Implementation of Evidence-based Asthma Interventions in PostKatrina New Orleans: The Head-off Environmental Asthma in Louisiana (HEAL) Study. Environ Health Perspect. 2012;120(11):1607-1612. doi: 10.1289/ehp.1104242.

22. Grimsley LF, Chulada PC, Kennedy S, White L, Wildfire J, Cohn RD, et al. Indoor environmental exposures for children with asthma enrolled in the HEAL study, post-Katrina New Orleans. Environ Health Perspect. 2012;120(11):1600-1606. doi: 10.1289/ehp.1104840.

23. National Surveillance of Asthma: United States, 2001-2010. P3 (Current Asthma Prevalence and Asthma Attack Prevalence).

24. Saporta D. Changes in Peak Flow Value During Immunotherapy Administration. J Environ Public Health. 2012;2012:212867. doi: $10.1155 / 2012 / 212867$. 